\title{
An Overview of Current and Future Vehicular Communication Technologies
}

\author{
Ádám Knapp ${ }^{1 *}$, András Wippelhauser¹, Dániel Magyar¹, Győző Gódor ${ }^{1}$ \\ ${ }^{1}$ Department of Networked Systems and Services, Faculty of Electrical Engineering and Informatics, Budapest University of \\ Technology and Economics, H-1111 Budapest, Müegyetem rkp. 3., Hungary \\ * Corresponding author, e-mail: knapp@hit.bme.hu
}

Received: 12 March 2020, Accepted: 12 March 2020, Published online: 03 August 2020

\begin{abstract}
The present paper provides a technical overview about the most relevant vehicular communication technologies including IEEE $802.11 \mathrm{p}$, IEEE 802.11 bd, LTEV2X and NRV2X. IEEE 802.11p is the most matured one Wi-Fi based technology, and its successor, the IEEE $802.11 \mathrm{bd}$ is expected to be released in 2021. As CV2X (Cellular Vehicle to Everything) technologies, LTEV2X (Long Term Evolution V2X) and NRV2X (New Radio V2X) are discussed in this paper. The former one is already available, while the latter one's final specification will be finalized in 2020 by the 3GPP (Third Generation Partnership Project). These four technologies also motivate the already started transformation of vehicle industry by enabling basic safety features and more efficient traffic management, as well as cooperative maneuver execution towards high-level automated driving. The comparison of these technologies is essential to clarify their benefits completely. These technologies are considered as competitors, however, it is expected that they will coexist in the same geographical region. Thus, they will share on the same unlicensed frequency bands in $5.9 \mathrm{GHz}$ domain. Therefore, different coexistence scenarios are also discussed in the paper to see how their advantages could be utilized.
\end{abstract}

Keywords

intelligent transportation system, NRV2X, LTEV2X, IEEE 802.11p, IEEE 802.11bd

\section{Introduction}

In the recent decades, the ICT sector has been gone through a remarkable evolution and it infiltrated in each aspect of our lives. That also includes the automotive industry, where the safety of passengers and other participants of transport are crucial. The vehicular communication technologies incorporate a new level of cooperation between the aforementioned industries. They promise not just safe, but more efficient and comfortable transport towards the fully automated and connected vehicles.

In the last ten years, significant efforts have been put in the development of V2X (Vehicular to Everything) communication technologies. Thanks to several European Union funded projects (C-Roads, 2020) more and more roads are equipped with communication units, as well as car manufacturers have noticed the importance of such technology and started to integrate communication devices in their cars (Butler, 2019; Toyota, 2018; Volkswagen, 2017).

The two major groups of vehicular communication technologies that are discussed in this paper are the WiFi based and Cellular V2X (CV2X) based solutions. The former one is mostly referred as the IEEE $802.11 \mathrm{p}$, but soon its successor will be also available known as IEEE $802.1 \mathrm{lbd}$. The latter group includes the Long Term Evolution V2X (LTEV2X) specified as part of the fourth generation and the New Radio V2X (NRV2X) as part of the fifth generation mobile communication standard elaborated by the Third Generation Partnership Project (3GPP).

The IEEE $802.11 \mathrm{p}$ is released in 2010 , therefore, its relatively long history includes numerous simulation and field trial results worldwide. The LTEV2X is more recent, thus, it applies advanced solutions in its physical layer (PHY) compared to the IEEE 802.11p, but its performance, reliability and scalability is not completely verified. Contrarily, the other two technologies are still not fully specified, but many details have already been available. In general, it can be stated that the new V2X technologies take over many sophisticated features from the state-of-the-art wireless communication technologies, i.e. from WiFi 5/6 and from New Radio, like numerology (changeable subcarrier spacing), 256-QAM modulation 
scheme, Low-Density Parity-Check (LDPC) codes and MIMO (Multiple Input Multiple Output).

This paper is structured as follows. Section 2 presents the four aforementioned vehicular communication technologies organized into Subsections 2.1 to 2.4, while Subsection 2.5 contains the comparison of them and the related discussion. Section 3 surveys and investigates relevant coexistence scenarios. Finally, Section 4 concludes the paper.

\section{Overview of vehicular communication technologies}

Section 2 describes the four relevant, WiFi and cellular based V2X communication technologies and compares them along their main parameters. To be more specific, the presented technologies implement the physical and the data link layers according to the Open Systems Interconnection model of International Organization for Standardization (ISO/OSI 7-layer model) (International Organization for Standardization / International Electrotechnical Comission, 1994). The physical layer is responsible for transmitting and receiving the raw data between a communication equipment and a physical transmission medium. The PHY transforms the data represented as bits into radio signals and vice versa. The specifications of PHY of a wireless equipment define characteristics like channel access methods, maximum transmission distances, modulation schemes, channel coding methods, signal timing and frequency, etc. These parameters determine the physical data rates.

The data link layer provides direct data transfer, i.e. a link, between two communicating devices. Furthermore, it is responsible for error detection and/or correction capabilities, i.e. it is able to detect and possibly correct errors originating from the physical layer. This layer is often divided into Logical Link Control (LLC) and Medium Access Control (MAC) sublayers. The main purpose of LLC is to identify and encapsulate upper, i.e. network layer protocols, and to control error checking and frame synchronization, while the MAC controls how the nodes of a network gain access to a medium and permission to transmit data.

Nevertheless, the V2X communication and the related standards are organized into a complex system that is slightly different depending on the geographical regions (Jakó et al., 2019). This paper focuses on the Cooperative Intelligent Transport Systems (CITS) protocol architecture and the ITSG5 protocol stack defined by the European Telecommunications Standards Institute (ETSI). In this general architecture the Radio Access Technology (RAT) is not specified, thus each discussed V2X communication technology in the present paper is a candidate to transmit the data originating from higher layer protocols and from the applications. Therefore, the evaluation of these technologies has to take into consideration their role in this complex system, as well as the possible V2X applications with their characteristics.

\subsection{IEEE 802.11p}

The IEEE $802.11 \mathrm{p}$ was released in 2010 by the IEEE, thus it is the oldest and most used V2X communication technology nowadays. It is often referred as Wireless Access in Vehicular Environments (WAVE) or Dedicated ShortRange Communications (DSRC). The IEEE 802.11p is an amendment of the traditional IEEE 802.11, more specifically its base is the IEEE $802.11 \mathrm{a}$ version. It inherits the Orthogonal Frequency Division Multiplexing (OFDM)based PHY, but the operation frequency slightly shifted to $5.9 \mathrm{GHz}$ from $5 \mathrm{GHz}$, where spectrum with $10 \mathrm{MHz}$ channels is allocated worldwide exclusively for V2X communication. This means that the subcarrier spacing and bandwidth are halved that result in doubled symbol duration in case of IEEE 802.11p compared to IEEE 802.11a. Besides that, the duration of Cyclic Prefix (CP) was also increased to two times longer in order to offset larger delay spreads and make it more adequate for real mobile environment. This setup of OFDM leads to $8 \mu$ s long symbol time and $156.25 \mathrm{kHz}$ wide subcarrier spacing with 52 subcarriers. Like similar modern communication systems, the IEEE 802.11p uses adaptive Modulation and Coding Scheme (MCS) including Binary and Quadrature Phase Shift Keying (B/QPSK), 16- and 64-Quadrature Amplitude Modulation (QAM) schemes with Binary Convolutional Coding (BCC). The standard allows eight different MCS (IEEE Standard for Information Technology, 2016), and the actual MCS used for communication is selected according to the instantaneous quality of the wireless channel. Note that, each other presented technology provides such mechanism as well, however, the modulation types and coding methods are slightly different.

Furthermore, IEEE 802.11p PHY is divided into two sublayers, and reuses the same reference and preamble signal pattern for channel estimation and synchronization described by IEEE 802.11a. The Physical Medium Dependent (PMD) sublayer is considered as an interface to the physical transmission medium. Meanwhile the Physical Layer Convergence Protocol (PLCP) sublayer is responsible for communicating with the MAC layer and transforming the Packet Data Unit (PDU) from the MAC 
layer to an OFDM frame. The PLCP Packet Data Unit (PPDU) contains a preamble and signal field and a payload part that carries the useful data. The preamble indicates the beginning of the physical frame. It is applied for selecting the proper antenna and correcting the frequency and timing offset. The preamble field carries training symbols: ten of that are short and are used for establishing automatic gain control, diversity selection and the coarse frequency offset estimate of the carrier signal; the remaining two are long training symbols that are applied by the receiver for channel and fine frequency compensation estimation.

Based on the listed characteristics, IEEE 802.11p allows transmission rates from 3 to $27 \mathrm{Mbps}$, and the outdoor transmission distance is at most $1000 \mathrm{~m}$ depending on the geographical environment.

Finally, the operation of MAC layer was also adapted to the specific needs of vehicular communication. The MAC still applies Carrier Sense Multiple Access with Collision Avoidance (CSMA/CA), but there is no exponential back-off, like in case of the original WiFi medium access mechanism, i.e. the parameter Contention Window (CW) remains fixed in order the avoid large $\mathrm{CW}$ sizes that could lead to high latencies. In addition, due to the broadcast-based application of V2X, there is no acknowledgement mechanism implemented in IEEE 802.11p.

\subsection{IEEE 802.11bd}

As new CITS applications emerge and their usage field start to shift from the reactive domain, like notification based perception and basic safety applications, to the proactive domain, e.g. cooperative maneuver execution, IEEE $802.11 \mathrm{p}$ is not able to cope with the increasing demands such as higher throughput, lower and constant latency, as well as with the increasing traffic density. Thus, the IEEE started to develop a new, improved variant of IEEE 802.11p known as IEEE 802.11bd in 2018. Its expected release date is 2021; however, its main features are already foreseen.

Beside the mentioned demands, several IEEE 802.11p related requirements have been defined including interoperability, coexistence, fairness and backward compatibility (Sun, 2019b).

As the basis of IEEE 802.11p is the IEEE 802.11a, the characteristics of IEEE $802.11 \mathrm{bd}$ originates from IEEE $802.11 \mathrm{n} / \mathrm{ac} / \mathrm{ax}$ also known as WiFi 4/5/6 according to the new naming conventions. Thanks to this, IEEE $802.11 \mathrm{bd}$ is prepared to operate on both 10 and
$20 \mathrm{MHz}$ bandwidth. However, this inheritance brings several design decisions as well, such as the application of midambles. Its function and form are similar to the preamble except for their location within the frame. The preambles are located at the starting of the frame (PPDU), and are used for initial channel estimation, while the midambles are inserted inside a frame to provide continuous channel estimation. The real benefit of midambles come forth in fast-varying radio channel that is typical in vehicular environment. It is envisaged that the periodicity of midamble insertion will probably depend on modulation, Doppler spread and other similar parameters. Note that the DeModulation Reference Signal (DMRS) symbols play similar role in CV2X systems.

Furthermore, to increase the reliability of the communication, IEEE $802.11 \mathrm{bd}$ will support one or more retransmission of a message. Like other state-of-the-art wireless communication technologies, numerology of OFDM is introduced as well. It allows to change the subcarrier spacing and by that the symbol duration according to the radio environment (Rui et al., 2018). The possibilities are 2, 4, 8 times down-clock with 64, 128, 256 subcarriers resulting in 312.5, 156.25 and $78.125 \mathrm{kHz}$ subcarrier distances respectively.

Another new feature is the Dual Carrier Modulation (DCM) technique originating from IEEE 802.11ax. It takes advantages of frequency diversity by transmitting same symbols twice over sufficiently far-apart subcarriers (Dongguk et al., 2019). Consequently the modulation order have to be increased (e.g. from BPSK to QPSK, or from QPSK to 16-QAM) to achieve the same throughput. Regardless of the modulation order increase, DCM is capable of improving the block-error-rate (BLER) performance. Additionally, DCM has been implemented for range extension in IEEE 802.11ax that can be similarly utilized in case of IEEE $802.11 \mathrm{bd}$ as well.

As general characterization of the PHY of IEEE 802.11bd, it will use B/QPSK, 16-, 64- and 256QAM modulations with LDPC codes resulting in ten MCS. Furthermore, multiple antennas will be applicable at both the receiver and transmitter side for spatial diversity or spatial multiplexing to increase the reliability or the throughput (Hongyuan et al., 2018).

Finally, there are further improvements foreseen in IEEE 802.11bd such as multi-channel operations (MCO) and usage of millimeter wave (mmWave) frequency bands that are not discussed here. More details are available in Naik et al. (2019). 


\subsection{LTEV2X}

One of the CV2X technologies is the LTEV2X that has been specified in Release 14 by the 3GPP. It provides Device-to-Device (D2D) transmission using the LTE's radio interface. D2D or Sidelink communication is part of earlier Release 12, and defines a new interface that is called PC5, besides the original LTEUu interface. PC5 has four different Transmission Modes (TM). From vehicular communication's point of view, Mode 3 and 4 are relevant. These modes introduce low-latency data transfer, improved support for higher velocity and a new distributed channel access mechanism. Mode 3 delegates the resource allocation to the cellular network, i.e. to the eNodeB, thus cellular coverage is necessary in this case. Contrarily, in Mode 4 the devices autonomously choose their radio resources using a distributed scheduling technique supported by congestion control methods. Therefore, Mode 4 is usually considered the baseline mode, and it makes LTE-V2X as another option to IEEE 802.11p. To avoid the high PAPR of the OFDM, LTEV2X reuses the LTE's uplink communication scheme known as Single-Carrier Frequency-Division Multiple Access (SCFDMA), and supports adaptively QPSK, 16- and 64-QAM modulations (the latter one is introduced in Release 15) with turbo coding. These are inherited from the normal LTE radio interface, as well as the $15 \mathrm{kHz}$ subcarrier spacing that provides even better robustness against channel distortions in mobile environment compared to the $156.25 \mathrm{kHz}$ of the IEEE 802.11p. Furthermore, the granularity of radio resources is also higher allowing more flexible resource allocation. Altogether, the PHY of LTEV2X is more advanced compared to IEEE 802.11p.

\subsection{NRV2X}

The cellular standardization activities did not stopped at the LTE, 3GPP released the fifth generation mobile communication technology in 2019 known as New Radio. The Release 15 defines a new radio interface for the wireless communication, as well as it considers different application areas, such as enhanced Mobile Broadband (eMBB) and Ultra Reliable Low Latency Communications (URLLC), and use cases like V2X communication. However, this release extensively specifies the eMBB, URLLC and vehicular communication related details are expected in Release 16.

Note that the NRV2X is designed to supplement LTEV2X not to replace it by allowing such V2X applications that are not supported by LTEV2X. It is envisaged that these two will coexist and newer vehicles will be equipped with both technology (Vodafone, 2018).
According to the philosophy of $5 \mathrm{G}$ mobile network, NRV2X is designed to cover a wide range of transmission requirements. It supports $\mathrm{V} 2 \mathrm{X}$ applications with varying degrees of throughput, latency and reliability demands such as use cases requiring transmission of periodic traffic, or reliable delivery of aperiodic messages. To provide more flexible usage two new communication types, i.e. unicast and groupcast, will be introduced in NRV2X. Furthermore, the utilization of mmWave bands are also considered, however, this feature is expected to be elaborated in later releases (probably in Release 17).

Like LTEV2X, NRV2X operates on sidelink, and there are two modes envisaged. NRV2X Mode 1 and 2 have the same characteristics as LTEV2X Mode 3 and 4. In Mode 1, the resource allocation is the responsibility of gNodeB, thus cellular coverage is required. However, the communication takes place directly between the devices. Contrarily, no gNodeB coverage is necessary for Mode 2. It will provide similar mechanisms for resource allocation than LTEV2X. Furthermore, Mode 2 will have several sub-modes. They are not completely designed, but the aim of such distribution of functionality is the higher flexibility required by wide range of $\mathrm{V} 2 \mathrm{X}$ use cases.

Regarding the physical layer of NRV2X, it inherits its features from New Radio with V2X specific enhancements. NR supports both OFDM (for high throughput efficiency) and Discrete Fourier Transform spread OFDM (DFTsOFDM) (for low link budget devices) for uplink transmissions. It is capable of operating two frequency ranges: sub-6 GHz and mmWave (this will be further elaborated in later releases as mentioned). It also utilizes the benefits of scalable OFDM numerology. Thus, 15, 30 and $60 \mathrm{kHz}$ subcarrier spacing are defined for sub-6 $\mathrm{GHz}$, while 60 and $120 \mathrm{kHz}$ are specified for frequency bands higher than $6 \mathrm{GHz}$. The use of higher subcarrier spacings helps reducing latency. In addition, the frame structure is also improved compared to LTE that results in an even more flexible resource allocation. In New Radio a slot contains 14 OFDM symbols, but a sub-frame includes different number of slots depending on the subcarrier spacing. Radio resources can be dedicated per slot unlike LTE, where the minimum Transmission Time Interval (TTI) is one sub-frame, i.e. one millisecond. For further latency reduction, NR also enables to transmit data using only 2, 4 or 7 OFDM symbols, called as mini-slot, without any slot boundaries. It is expected that multiple antennas will be applicable at both the receiver and transmitter side for spatial diversity or spatial multiplexing to increase the reliability or the throughput. 
Finally, NRV2X supports QPSK, 16-QAM, 64-QAM modulation schemes with LDPC codes for user data transfer and Cyclic Redundancy Check (CRC) assisted polar codes for control data transmission.

\subsection{Comparison of radio access technologies}

To conclude Subsection 2.1-2.4, Table 1 lists the characteristics of the presented V2X technologies. The necessity of these communication solutions is unquestionable to achieve much safer and efficient transportation system. However, the limitations of IEEE 802.11p are already reached particularly in high density traffic situations, as well as by the introduction of newer and newer V2X applications. Even though, LTEV2X is more advanced from both PHY and MAC aspects, it is also not able to cope with the increased demands by itself. These two already available technologies are perfectly fit for basic safety applications. Nevertheless, the next generation communication solutions, i.e. NRV2X and IEEE $802.11 \mathrm{bd}$ will enable more advanced applications towards fully automated vehicles, such as cooperative maneuver execution. Note that there are still some open questions regarding the details, since these are not completely specified at the time writing of this paper.

\section{Coexistence scenarios of $\mathrm{V} 2 \mathrm{X}$ communication technologies}

Section 3 describes different coexistence scenarios of vehicular communication technologies. The most relevant scenarios are grouped into subsections and discussed separately.

\subsection{Coexistence of IEEE 802.11p and IEEE 802.11bd}

As mentioned, there are requirements that have to be taken into account during the design of IEEE 802.11bd (Sun, 2019b):

- interoperability, i.e. IEEE 802.11 p capable radio transceivers have to be able to recognize (minimum one mode of) transmissions from 802.11bd equipment, and vice-versa;

- fairness, i.e. IEEE 802.11bd and its predecessor have to get equal channel access possibilities in co-channel scenarios;

- coexistence, i.e. IEEE 802.11 bd have to be capable of recognizing IEEE 802.11p transmissions and postpone channel access, and vice-versa;

- backward compatibility, i.e. minimum one mode of IEEE $802.11 \mathrm{bd}$ have to be interoperable with IEEE 802.11p.

Of course, these requirements affect each other, and the details of the operation of IEEE 802.11bd are still under discussion. Nevertheless, it seems obvious that in such cases when devices using both WiFi based technologies operate co-channel in the same geographical area, IEEE 802.11bd equipment should send all frames according to IEEE 802.11p frame structure. Thus, a method have to be elaborated for an IEEE 802.11 bd device to be able to advertise its capabilities to other IEEE 802.11bd devices. Otherwise, it would happen that even after there is no IEEE 802.11p equipment nearby, IEEE $802.11 \mathrm{bd}$ devices continue to transmit messages in IEEE 802.11p frame format. A proposed solution

Table 1 Comparison of V2X communication technologies

\begin{tabular}{|c|c|c|c|c|}
\hline Feature & IEEE $802.11 p$ & IEEE $802.11 \mathrm{bd}$ & LTEV2X & NRV2X \\
\hline Radio bands of operation & $5.9 \mathrm{GHz}$ & $5.9 \mathrm{GHz}$ and $60 \mathrm{GHz}$ & $5.9 \mathrm{GHz}$ & $5.9 \mathrm{GHz}$ \\
\hline Operation bandwidth & $10 \mathrm{MHz}$ & $10 / 20 \mathrm{MHz}$ & Flexible: $1.4 / 5 / 10 / 20 \mathrm{MHz}$ & $\begin{array}{c}\text { Flexible: } \\
\text { sub-6 GHz: } \max .100 \mathrm{MHz} \\
\text { mmWave: } \max .400 \mathrm{MHz}\end{array}$ \\
\hline Waveform & OFDM & OFDM & SCFDMA & OFDM/DFTsOFDM \\
\hline Subcarrier spacing & $156.25 \mathrm{kHz}$ & $\begin{array}{c}78.125 \mathrm{kHz}, 156.25 \mathrm{kHz} \\
312.5 \mathrm{kHz}\end{array}$ & $15 \mathrm{kHz}$ & $\begin{array}{c}\text { sub-6 GHz: } 15,30,60 \mathrm{kHz} \\
\text { mmWave: } 60,120 \mathrm{kHz}\end{array}$ \\
\hline Supported modulations & $\begin{array}{c}\text { BPSK, QPSK, 16-QAM, } \\
\text { 64-QAM }\end{array}$ & $\begin{array}{c}\text { BPSK, QPSK, 16-QAM, } \\
\text { 64-QAM, 256-QAM }\end{array}$ & $\begin{array}{l}\text { Rel. 14: QPSK, 16-QAM } \\
\text { Rel. 15: 64-QAM }\end{array}$ & QPSK, 16-QAM, 64-QAM \\
\hline Channel coding & $\mathrm{BCC}$ & LDPC & Turbo codes & $\begin{array}{c}\text { Data: LDPC } \\
\text { Control: Polar with CRC }\end{array}$ \\
\hline Number of MCS & 8 & 10 & $>20$ & $>20$ \\
\hline Spatial streams & One & Multiple & Multiple & Multiple \\
\hline Re-transmission & None & Congestion dependent & Blind & HARQ \\
\hline Channel estimation & Preambles only & Preambles and midambles & DMRS: 4/sub-frame & DMRS: flexible \\
\hline Comm. types (PHY layer) & Broadcast & Broadcast & Broadcast & $\begin{array}{c}\text { Broadcast, Groupcast, } \\
\text { Unicast }\end{array}$ \\
\hline
\end{tabular}


to avoid such situations is to use a flag at MAC layer level that indicates the availability of IEEE $802.11 \mathrm{bd}$ to other IEEE 802.11bd capable devices (Sun, 2019a).

\subsection{Coexistence of LTEV2X and NRV2X}

The coexistence between IEEE 802.11p and IEEE 802.11bd can be considered to be solved, because IEEE $802.11 \mathrm{bd}$ is designed to be coexist and backward compatible with IEEE 802.11p. However, the approaches for the coexistence of NRV2X and LTEV2X are different, since the backward compatibility between the two CV2X technologies is not available; therefore only non-co-channel coexistence scenarios are considered (Vodafone, 2018). Two possible scenarios were reported in Qualcomm Inc. (2018). The Frequency Division Multiplexing scenario is advantageous because the time synchronization is not necessary between the two radio modules. The drawback of FDM is that if LTEV2X and NRV2X modules operate at close bands, their transmission will likely impair the reception of the other module. The FDM based approach can also face regulatory transmission restrictions, if both modules operate in the $5.9 \mathrm{GHz}$ band. The issues related to these restrictions might be handled with the prioritization of the radio modules. On the other hand, the Time Division Multiplexing (TDM) scenario prohibits transmission on the two radio modules simultaneously using strict time synchronization. This solution also uses two separate channels. Thus, its benefit is to enable the maximum acceptable transmission power on each radio modules, but it introduces additional latency that is not acceptable in some use cases. If using adjacent channels, the half-duplex problem is also present in the TDM approach.

\subsection{Coexistence of LTE and WiFi}

For the extensive investigation, it is worth to take a look on the already existing coexistence solutions between LTE and WiFi.

In Naik et al. (2018), the authors provided a comprehensive survey of the possible coexistence scenarios in the $5 \mathrm{GHz}$ bands. There are many different wireless technologies, which operate in this frequency band, e.g. LTE, WiFi, radar systems, Dedicated Short Range Communication (DSRC) system, and these technologies can interfere each other especially if the used bands overlap or are the same.

Since the channel access methods used by WiFi and LTE are fundamentally different, the fairness of channel usage in the unlicensed frequency bands by Wi-Fi devices could be harmed. LTE is a cellular technology, thus the scheduling is centralized and controlled by base stations, i.e. eNodeBs; however, in Wi-Fi networks the channel access method is distributed. In the LTE case, the eNodeB defines which equipment can use the channel and when. Contrarily, in WiFi case, each device has to listen the channel before starting a transmission. As a result, it is expected that when WiFi based technology and unlicensed LTE operate within the same ITS frequency band, LTE will shrivel WiFi capable devices. In Cavalcante et al. (2013) and in Zhang et al. (2015), the authors showed by simulations that the WiFi system throughput can be degraded by up to $68 \%$, if an LTE system also operates in the same frequency.

There are two categories of LTE methods that operate in unlicensed frequency band. One is the license-anchored, and the other is the non license-anchored method. In the former case, such as LTE-Unlicensed (LTEU) and Licensed Assisted Access (LAA), the primary carrier, referred to as the anchor, uses licensed portions of the spectrum. The secondary carriers can operate in the $5 \mathrm{GHz}$ unlicensed spectrum. In this case, the control channels and data that require certain QoS parameters are transmitted on the primary carrier. However, in unlicensed non licensed-anchor systems, both control and data traffic are carried by the unlicensed frequency.

Currently, four different LTE technologies exist that are able to operate in the $5 \mathrm{GHz}$ unlicensed bands.

LTE in Unlicensed Spectrum or LTE-Unlicensed is proposed by Qualcomm and Ericsson in 2013. LTEU relies on 3GPP Release 10-12 functionality, with specifications defined by the LTEU Forum. This technology does not support the so-called Listen-Before-Talk (LBT) mechanism.

License Assisted Access (LAA) was standardized in 3GPP Release 13. Contrarily to LTEU, LAA is expected to use the LBT method, since it required by regulatory organizations in Europe and Japan. More alignment is necessary in licensed LTE compared to LTEU systems to satisfy this demand for LBT.

LTEWLAN Aggregation (LWA) was approved as an LTEWLAN Radio Level Integration and Interworking Enhancement in 2015, and it was standardized in 3GPP Release 13 in 2016. Like the previous ones, LWA is a licensed-anchor based system, as well. Nevertheless, this technology uses the WiFi PHY and MAC mechanisms for LTE data transmissions. Thus, the aforementioned fairness issues between LTE and WiFi is handled.

MulteFire is more recent LTE feature, it does not require an "anchor" in the licensed spectrum. The first specification was released in 2017, which is based on 3GPP Releases 13 (for downlink) and 14 (for uplink). This technology uses such a protocol for accessing medium that is based on the LBT scheme. 


\subsection{Coexistence of CV2X and WiFi based technologies}

The physical layers of WiFi based, i.e. ITS-G5, and CV2X technologies are different, as well as they applies different medium access control protocols, thus the operation of them in the $5.9 \mathrm{GHz}$ frequency band at the same geographic region would result in mutual harmful co-channel interference. In order to reduce this interference in short term, a possible solution is adjacent channel sharing by the definition of so-called preferred channels to ITSG5 (5895$5905 \mathrm{MHz})$ and CV2X (5875-5885 MHz). This simple spectrum sharing method proposed by $5 \mathrm{G}$ Automotive Association (2018) is based on technology recognition and dynamic ITS channel selection. The implementation of the proposed technique contains three steps. In every step, each of WiFi based and CV2X systems are able to support safety-related ITS services without interfering each other. The amount of usage of the spectrum resource is the main difference between the steps. The biggest advantage of this solution is the simplicity, but the proposed detect-andvacate method has to be further elaborated and specified.

\section{Conclusion}

In this paper, the current and future V2X communication technologies were overviewed and the most recent information of the latter ones was summarized. Then, they were compared along their main parameters. Since the

\section{References}

Butler, D. (2019) "How 'Talking' and 'Listening' Vehicles Could Make Roads Safer, Cities Better", City of Tomorrow, [online] 07 January 2019. Available at: https://medium.com/cityoftomorrow/howtalking-and-listening-vehicles-could-make-roads-safer-cities-better-f215c68f376f [Accessed: 11 February 2020]

Cavalcante, A. M., Almeida, E., Vieira, R. D., Choudhury, S., Tuomaala, E., Doppler, K., Chaves, F., Paiva, R. C. D., Abinader, F. (2013) "Performance Evaluation of LTE and Wi-Fi Coexistence in Unlicensed Bands", In: 2013 77th Vehicular Technology Conference (VTC Spring), Dresden, Germany, pp. 1-6. https://doi.org/10.1109/VTCSpring.2013.6692702

C-Roads "C-Roads - The Platform of harmonized C-its deployment in Europe", [online] Available at: https://www.c-roads.eu/ [Accessed: 11 February 2020]

Dongguk, L., Jang, I., Park, E., Kim, S., Kim, J., Choi, J. (2019) "IEEE 802.11-19/0009r0: Consideration on features for 11bd", IEEE, St. Louis, Missouri, USA.
IEEE $802.11 \mathrm{p}$ is a decade old, it is the most investigated system; however, the LTEV2X was released in the recent years and got in the focus. It has more advanced PHY and MAC layers compared to IEEE 802.11p, but it has its limitation due to the SPS scheme in Mode 4, while Mode 3 requires coverage by an eNodeB. It has to be mentioned that although the standard is available, it is not yet widely deployed in practice. The two future technologies promise higher performance compared to both the IEEE 802.11p and the LTEV2X that will enable higher-level cooperation of traffic participants and of the infrastructure.

Note that the discussed technologies are often compared suggesting that they are competitors. However, they will eventually coexist, and must be considered as complementary systems, thus all of them have to be used according their benefits in our opinion. Therefore, as future work, the coexistence of these technologies has to be further elaborated to be able to fully utilize the potential of CITS applications.

\section{Acknowledgement}

The authors would like to thank László Bokor and Norbert Varga for their advices and comments to the present paper.

The research reported in this paper was supported by the Higher Education Excellence Program in the frame of Artificial Intelligence research area of Budapest University of Technology and Economics (BME FIKP-MI/FM).

Hongyuan, Z., Rui, C., Yan, Z., Liwen, C., Jinjing, J., Hui-Ling, L., Manish, K., Sudhir, S., Lepp, J., Montemurro, M., Amer, H., Takenori, S., Yukimasa, N., Jianlin, G., Jianhan, L., Bo, S., Nandagopalan, S., Hari, K., Zerod, R., Satish, P., Jing, M., Kentaro, I., Fumihide, K. (2018) "IEEE 802.11-18/0513r2: 802.11 for next generation V2X communication", IEEE, [online] Available at: https://mentor.ieee.org/802.11/dcn/18/11-18-051302-0wng-802-11-for-next-generation-v2x-communication.pptx [Accessed: 11 February 2020]

IEEE Standard for Information Technology (2016) "IEEE 802.11-2016 IEEE standard - part 11: Wireless LAN medium access control (MAC) and physical layer (PHY) specifications", IEEE Standard for Information Technology, Piscataway, NJ, USA.

International Organization for Standardization / International Electrotechnical Comission (1994) "ISO/IEC 7498-1:1994(E) Information technology - Open System Interconnection - Basic Reference Model: The Basic Model", International Organization for Standardization / International Electrotechnical Comission, Geneva, Switzerland. 
Jakó, Z., Knapp, Á., Nagy, L., Kovács, A. (2019) "Vehicular communication - a technical overview", In: Lu, M. (ed.) Cooperative Intelligent Transport Systems: Towards High-Level Automated Driving, The Institution of Engineering and Technology, London, UK, pp. 135-159. https://doi.org/10.1049/PBTR025E_ch7

Naik, G., Liu, J., Park, J. M. J. (2018) "Coexistence of Wireless Technologies in the $5 \mathrm{GHz}$ Bands: A Survey of Existing Solutions and a Roadmap for Future Research", IEEE Communications Surveys \& Tutorials, 20(3), pp. 1777-1798. https://doi.org/10.1109/COMST.2018.2815585

Naik, G., Choudhury, B., Park, J. M. (2019) "IEEE 802.11bd \& 5G NR V2X: Evolution of Radio Access Technologies for V2X Communications", IEEE Access, 7, pp. 70169-70184. https://doi.org/10.1109/ACCESS.2019.2919489

Qualcomm Inc. (2018) "R1-1812000, 3GPP TSG RAN WG1 94b: Summary of Coexistence Aspects in NR-V2X Study", Qualcomm Inc., Chengdu, China.

Rui, C., Zhang, H., Sharma, P. (2018) "IEEE 802.11-18/1553r0: Doppler Impact on OFDM Numerology for NGV", IEEE, Hanoi, Vietnam.

Sun, B. (2019a) "IEEE 802.11-19/0237r4: IEEE 802.11 TGbd Mar 2019 agenda", IEEE, Vancouver, Canada.

Sun B, Zhang H. (2019b) "IEEE 802.11-18/0861r9: 802.11 NGV proposed PAR", IEEE, Piscataway, NJ, USA. [online] Available at: https://mentor.ieee.org/802.11/dcn/18/11-18-0861-06-0ngv-ieee802-11-ngv-sg-proposed-par.docx [Accessed: 11 February 2020]
Toyota (2018) "Toyota and Lexus to Launch Technology to Connect Vehicles and Infrastructure in the U.S. in 2021", Toyota Newsroom, [online] 16 April 2018. Available at: https://pressroom.toyota.com/ toyota-and-lexus-to-launch-technology-connect-vehicles-infrastructure-in-u-s-2021/ [Accessed: 11 February 2020]

Vodafone (2018) "RP-181480: New SID: Study on NR V2X", In: Proceedings on 3GPP Planery Meeting, Sorrento, Italy, pp. 1-10.

Volkswagen (2017) "With the aim of increasing safety in road traffic, Volkswagen will enable vehicles to communicate with each other as from 2019", Volkswagen Newsroom, [online] 28 June 2017. Available at: https://www.volkswagen-newsroom.com/en/ press-releases/with-the-aim-of-increasing-safety-in-road-trafficvolkswagen-will-enable-vehicles-to-communicate-with-each-other-as-from-2019-1023 [Accessed: 11 February 2020]

Zhang, R., Wang, M., Cai, L. X., Zheng, Z., Shen, X., Xie, L. L. (2015) "LTE-unlicensed: The future of spectrum aggregation for cellular networks", IEEE Wireless Communications, 22(3), pp. 150-159. https://doi.org/10.1109/MWC.2015.7143339

5G Automotive Association (2018) "Coexistence of C-V2X and ITS-G5 at 5.9GHz", [pdf] 5G Automotive Association, Munich, Germany, Available at: https://5gaa.org/wp-content/uploads/2018/10/ Position-Paper-ITG5.pdf [Accessed: 11 February 2020] 